\title{
ENERGY EFFICIENT ROUTING PROTOCOLS FOR WIRELESS SENSOR NETWORKS-A REVIEW
}

\author{
Saritha L. R. ${ }^{1}$
}

\begin{abstract}
Energy efficiency, storage capacity and computing power are major considerations while designing wireless sensor network. Wireless sensor networks use battery-operated computing and sensing devices. Most sensor network applications require energy autonomy for the complete lifetime of the node, which may span up to several years. There are many routing protocols like: location based, multipath, data centric, mobility based, hierarchical routing, hybrid routing etc. Clustering is used to prolong the lifetime of the wireless sensor networks. Clustering is the process where sensing area is divided in groups to balance the energy level of sensor nodes known as clusters. In this paper presents the study of different energy efficient routing protocols of wireless sensor networks and compared them on various parameters
\end{abstract}

Keywords - Wireless sensor networks (WSNs), Low-Energy Adaptive Clustering Hierarchy (LEACH), Secure Positioning for Sensor Networks (SPIN), nodes, routing protocols, Base Station (BS).

\section{INTRODUCTION}

In these days, wireless sensor network emerging as a promising and interesting area. Wireless Sensor Networks (WSN's) are being used in surveillance, industrial monitoring, traffic monitoring, habitat monitoring, health care monitoring, air pollution monitoring, forest fire detection, land slide detection, water quality monitoring, natural disaster prevention, industrial monitoring, cropping monitoring, machine health monitoring and crowd counting etc. which calls for monitoring before taking an appropriate action. The WSN is built from a few to several hundreds or thousands of nodes, where each node is connected to one or sometimes several sensors. Each such sensor network node has typically several parts: a radio transceiver with an internal antenna or connection to an external antenna, a microcontroller, an electronic circuit for interfacing with the sensors and an energy source, usually a battery or an embedded form of energy harvesting.

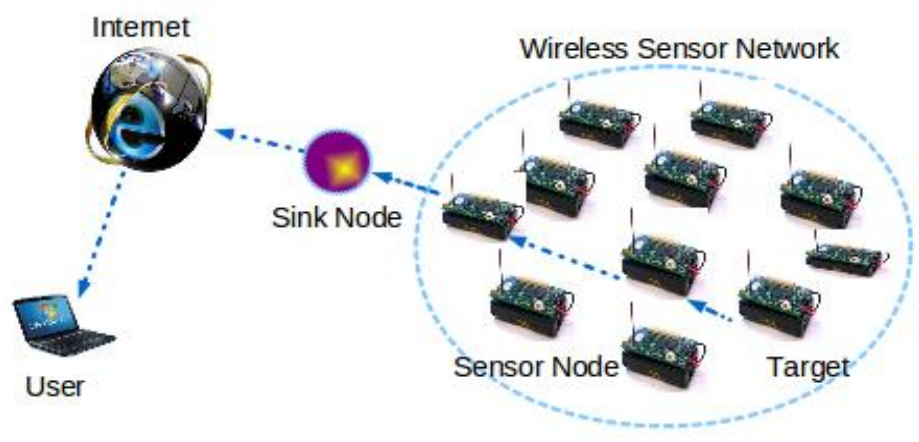

Figure: 1 Basic Architecture of Wireless Sensor network [3]

\footnotetext{
${ }^{1}$ Department of Information Technology SIES Graduate School of Technology, Nerul, Navi Mumbai
} 
Wireless sensor network can be categorized into two types: Unstructured WSN and Structured WSN. In unstructured WSN, the nodes are densely deployed and also the nodes can be deployed in ad-hoc manner in the sensing area or region. In Structured WSN Sensor node developments of some or all nodes are preplanned. The nodes placement is also planned. So, the maintenance of structured WSN is much easy as compare to Unstructured WSN.

\section{NETWORK DESIGN OBJECTIVES}

Most sensor networks are application specific and have different application requirements such as:

Small node size: Reducing node size can facilitate node deployment. It will also reduce the power consumption and cost of sensor nodes.

Low node cost: Reducing cost of sensor nodes is important and will result into the cost reduction of whole network.

Low power consumption: Since sensor nodes are powered by battery and it is often very difficult or even impossible to charge or recharge their batteries. It is important to reduce the power consumption of sensor nodes so that the lifetime of the sensor nodes, as well as the whole network is prolonged.

Reliability: Network protocols designed for sensor networks must provide error control and correction mechanisms to ensure reliable data delivery over noisy, error-prone, and time-varying wireless channels.

Scalability: Since the number sensor nodes in sensor networks are in the order of tens, hundreds, or thousands, network protocols designed for sensor networks should be scalable to different network sizes.

Self-configurability: Sensor nodes should be able to autonomously organize themselves into a communication network and reconfigure their connectivity in the event of topology changes and node failures.

Adaptability: In sensor networks, a node may fail, join, or move, which would result in changes in node density and network topology. Network protocols designed for sensor networks should be adaptive to such density and topology changes.

Channel utilization: Communication protocols designed for sensor networks should efficiently make use of the bandwidth to improve channel utilization, as sensor networks have limited bandwidth resources.

Fault tolerance: Sensor nodes should be fault tolerant and have the abilities of self testing, self-calibrating, selfrepairing, and self-recovering.

Security: A sensor network should introduce effective security mechanisms to prevent the data information in the network or a sensor node from unauthorized access or malicious attacks.

QoS support: Network protocol design should consider the QoS requirements of specific applications.

\section{TYPES OF ROUTING PROTOCOLS IN WSN}

(1) Location-based Protocols: MECN, SMECN, GAF, GEAR, Span, TBF, BVGF, GeRaF.

(2) Data-centric Protocols: SPIN, Directed Diffusion, Rumor Routing, COUGAR, ACQUIRE, EAD, Information-Directed Routing, Gradient- Based Routing, Energy-aware Routing, Information-Directed Routing, Quorum-Based Information Dissemination, Home Agent Based Information Dissemination.

(3) Hierarchical Protocols: LEACH, PEGASIS, HEED, TEEN, APTEEN.

(4) Mobility-based Protocols: SEAD, TTDD, Joint Mobility and Routing, Data MULES, Dynamic Proxy TreeBase Data Dissemination

(5) Multipath-based Protocols: Sensor-Disjoint Multipath, Braided Multipath, N-to-1 Multipath Discovery

(6) Heterogeneity-based Protocols: IDSQ, CADR, CHR

(7) QoS-based protocols: SAR, SPEED, Energy-aware routing

\section{A. LOCATION-BASED PROTOCOLS}

Sensor nodes are addressed by means of their locations. The distance between neighboring nodes can be estimated on the basis of incoming signal strengths. Relative coordinates of neighboring nodes can be obtained by exchanging such 
information between neighbors or by communicating with a satellite using GPS. To save energy, some location-based schemes demand that nodes should go to sleep if there is no activity.

\section{GEOGRAPHIC ADAPTIVE FIDELITY (GAF):}

It locates nodes in the network and makes the best use of them to have a better fidelity. All the nodes use a location identification technique to locate itself with its nearest neighbors by using location-information systems like GPS. In GAF, all the nodes arrange themselves according to grids also. All the nodes divide themselves in grids and all nodes which are under a same grid coordinate among themselves to see who will go into sleep state and for how long. Nodes in grid A can communicate with all the nodes in grid B that are adjacent. The time for sleeping is decided or depends on the application. GAF has three state states, discovery, active and sleeping. Every node starts with the discovery state. In this state the node turns on its radio and starts sending discovery messages. A node can fall into sleep state if there are other nodes in the grid which are equivalent in handling the fidelity before falling into the active state. In the active state the node sets a timeout value Ta which shows the remaining amount of time for which a node is intended to stay in active state. A node enters into sleeping state either from the discovery state or from the active state where Td is discovery time, Ta is active time and Ts is sleep time.

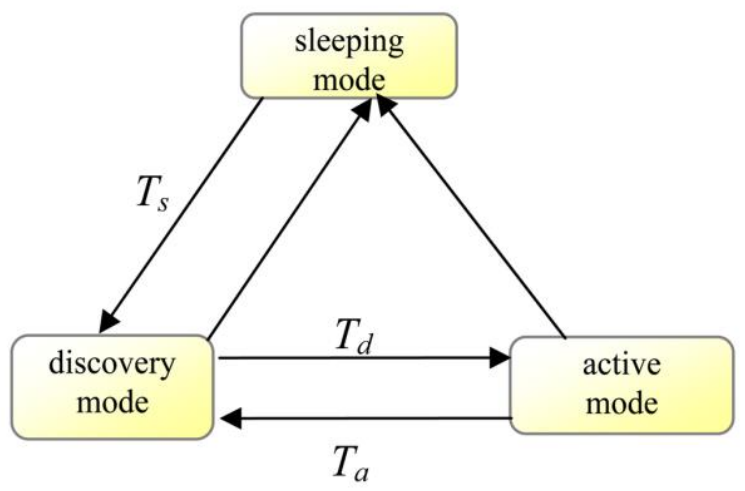

Figure 2: Three State Transitions in GAF

Depending on the Protocol Operation we can divide routing protocols as:

\section{MULTIPATH-BASED}

They use multiple paths rather than a single path in order to enhance network performance. For instance the fault tolerance can be increased by maintaining multiple paths between the source and destination at the expense of increased energy consumption and traffic generation.

\section{QUERY-BASED}

The destination nodes propagate a query for data from a node through the network; a node with this data sends the data that matches the query back to the node that initiated it.

\section{NEGOTIATION-BASED}

Use negotiation in order to eliminate redundant data transmissions. Communication decisions are also made based on the resources available.

\section{COHERENT-BASED}

The entity of local data processing on the nodes distinguish between coherent (minimum processing) and noncoherent (full processing) routing protocols

\section{QOS-BASED}

\section{(a) SPEED:}

The protocol provides three types of real-time communication services, namely, real-time unicast, real-time areamulticast and real-time area-anycast. SPEED is specifically tailored to be a stateless, localized algorithm with 
minimal control overhead. End-to-end soft real-time communication is achieved by maintaining a desired delivery speed across the sensor network through a novel combination of feedback control and non-deterministic geographic forwarding. SPEED is a highly efficient and scalable protocol for sensor networks where the resources of each node are scarce. When delivering data, the network balances between energy consumption and data quality through certain QoS metrics as delay, energy or bandwidth.

\section{(b) SAR (SEQUENTIAL ASSIGNMENT ROUTING)}

Sequential Assignment Routing (SAR) is the first of its kind which concentrates more on the energy efficiency and QOS factors. Creating multiple paths from the nodes to the sink helps in achieving a more energy efficient structure and also maximizes the fault tolerance of the network. Multiple paths are created in a tree structure, each rooted from the nearest neighbors' of the sink node. Each node tries to increase the tree or extend its roots by adding all those other neighbor nodes connecting the sink node. Nodes in the network which are low in energy reserves and which do not support real time factors like redundancy, bounded latency are deleted or ignored to be added as paths towards the sink. When a tree construction is completed we can see that every node has multiple paths from it through other nodes to reach some other node or to reach the sink. Using this structure, every node is capable of transmitting to all the other nearest single hop neighbors.

SAR maintains a path table which has all the best cost paths of the neighbor nodes. Whenever a node has to perform transmission, it checks for the best suitable and least cost path. SAR shows an optimized performance focusing on lowering of the energy consumption of each packet without considering its priority. A routing table update revolves around the network so as to update all the routing tables of the network in order to find out the depleted nodes in the network and ignore any further communication through the ruined path. SAR creates multiple trees where the roots of each tree is at one hop neighbor from the sink, A set of algorithms are used for performing organization, management and mobility management in the network so that it avoids overhead of the network traffic. SAR adapts quickly to node failures in the network, by using an handshaking procedure that enforces routing table consistency within the upstream and the downstream neighbor on each path, such that whenever there is an failure in the network the path table gets updated so that the new paths are elected to reduce traffic overheads and loss of data by utilizing more than the required energy.

\section{B. HIERARCHICAL PROTOCOLS}

Higher-energy nodes are used to process and send the information, while low-energy nodes are used to perform the sensing in the proximity of the target. The creation of clusters and assigning special tasks to cluster heads can greatly contribute to overall system scalability, lifetime, and energy efficiency. Hierarchical routing is an efficient way to lower energy consumption within a cluster, performing data aggregation and fusion in order to decrease the number of transmitted messages to the sink node.

A hierarchical approach breaks the network into clustered layers. Nodes are grouped into clusters with a cluster head that has the responsibility of routing from the cluster to the other cluster heads or base stations. Data travel from a lower clustered layer to a higher one. Although, it hops from one node to another, but as it hops from one layer to another it covers larger distances. This moves the data faster to the base station Clustering provides inherent optimization capabilities at the cluster heads.

\section{LOW-ENERGY ADAPTIVE CLUSTERING HIERARCHY (LEACH):}

LEACH is the first and most popular energy-efficient hierarchical clustering algorithm for WSNs that was proposed for reducing power consumption. $\mathrm{LEACH}$ is based on an aggregation technique that combines the original data into a smaller size of data that carry only meaningful information to all individual sensors. LEACH divides the a network into several cluster of sensors, which are constructed by using localized coordination and control not only to reduce the amount of data that are transmitted to the sink, but also to make routing and data dissemination more scalable and robust. LEACH uses a randomize rotation of high-energy $\mathrm{CH}$ position rather than selecting in static manner, to give a chance to all sensors to act as $\mathrm{CHs}$ and avoid the battery depletion of an individual sensor and die quickly. LEACH uses single-hop routing where each node can transmit directly to the cluster-head and the sink. Therefore, it is not applicable to networks deployed in large regions. While LEACH helps the sensors within their cluster dissipate their energy slowly, the CHs consume a larger amount of energy when they are located farther away from the sink. 
In LEACH algorithm each round is consist of two states:

(a) Setup State: during this step a cluster head is selected for that round.

(b) Steady State: In this phase the nodes send data to the cluster head.

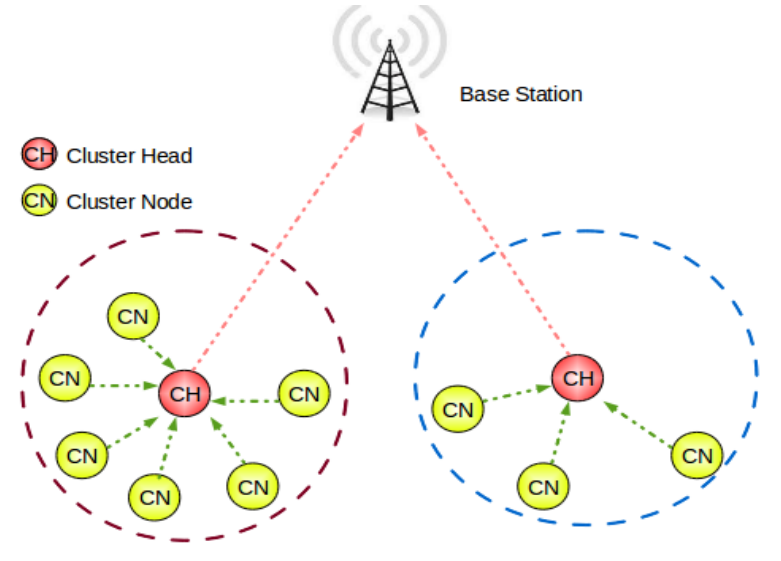

Figure 3: Illustration of LEACH Protoco

The major characteristics of this Protocol are as follows:

1. It rotates the cluster heads in a randomized fashion to achieve balanced energy consumption

2. Sensors have synchronized clocks so that they know the beginning of a new cycle,

3. Sensors do not need to know location or distance information.

There are some drawbacks of this protocol such as:

1. LEACH uses single-hop routing where each node can transmit directly to the cluster-head and the sink. Therefore, it is not applicable to networks deployed in large regions.

2. The idea of dynamic clustering brings extra overhead, e.g. head changes, advertisements etc., which may decrease the gain in energy consumption.

3. Random election of $\mathrm{CH}$, hence there is Possibility that all $\mathrm{CHs}$ will be concentrated in same area.

4. The protocol assumes that all nodes begin with the same amount of energy capacity in each election round, assuming that being a $\mathrm{CH}$ consumes approximately the same amount of energy for each node.

This protocol is most suited for constant monitoring such as monitor machinery for fault detection and diagnosis.

\section{POWER-EFFICIENT GATHERING IN SENSOR INFORMATION SYSTEMS (PEGASIS):}

PEGASIS is an extension of the LEACH protocol, which forms chains from sensor nodes so that each node transmits and receives from a neighbor and only one node is selected from that chain to transmit to the base station (sink). The data is gathered and moves from node to node, aggregated and eventually sent to the base station. The chain construction is performed in a greedy way. PEGASIS avoids cluster formation and uses only one node in a chain to transmit to the BS (sink) instead of using multiple nodes. A sensor transmits to its local neighbors in the data fusion phase instead of sending directly to its $\mathrm{CH}$ as in the case of LEACH. When a sensor fails or dies due to low battery power, the chain is constructed using the same greedy approach by bypassing the failed sensor.

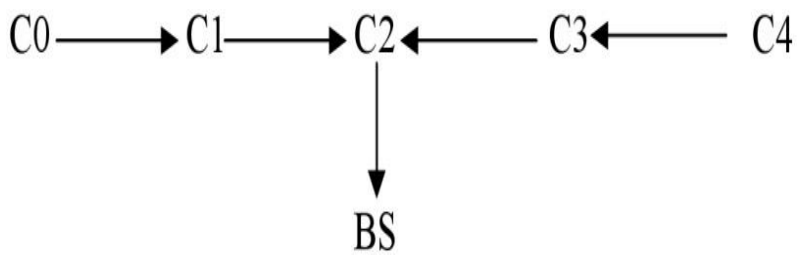

Figure 4: Chaining in PEGASIS 


\section{HYBRID ENERGY- EFFICIENT DISTRIBUTED CLUSTERING (HEED):}

HEED extends the basic scheme of LEACH by using residual energy and node degree as a metric for cluster selection to achieve power balancing. It operates in multi-hop networks, using an adaptive transmission power in the interclustering communication. HEED was proposed with four primary goals namely

(i) prolonging network lifetime by distributing energy consumption

(ii) terminating the clustering process within a constant number of iterations

(iii) minimizing control overhead, and

(iv) Producing well distributed $\mathrm{CHs}$ and compact clusters.

In HEED, the proposed algorithm periodically selects $\mathrm{CHs}$ according to a combination of two clustering parameters. The primary parameter is their residual energy of each sensor node and the secondary parameter is the intra-cluster communication cost as a function of cluster density or node degree (i.e. number of neighbors). The primary parameter is used to select an initial set of CHs while the secondary parameter is used for breaking ties. The HEED clustering improves network lifetime over LEACH clustering because LEACH randomly selects $\mathrm{CHs,} \mathrm{which} \mathrm{may} \mathrm{result} \mathrm{in} \mathrm{faster}$ death of some nodes.

The important features of this protocol are as follows:

1. HEED distribution of energy extends the lifetime of the nodes within the network thus stabilizing the neighboring node.

2. HEED does not require special node capabilities, such as location-awareness

3. HEED does not make assumptions about node distribution. The nodes also automatically update their neighbor sets in multi-hop networks by periodically sending and receiving messages. It operates correctly even when nodes are not synchronized.

4. The nodes only require local (neighborhood) information to form the clusters .

\section{THRESHOLD SENSITIVE ENERGY EFFICIENT SENSOR NETWORK PROTOCOL (TEEN):}

TEEN is a hierarchical clustering protocol, which groups sensors into clusters with each led by a $\mathrm{CH}$. The sensors within a cluster report their sensed data to their $\mathrm{CH}$. The $\mathrm{CH}$ sends aggregated data to higher level $\mathrm{CH}$ until the data reaches the sink. Thus, the sensor network architecture in TEEN is based on a hierarchical grouping where closer nodes form clusters and this process goes on the second level until the BS (sink) is reached. TEEN uses a data-centric method with hierarchical approach.

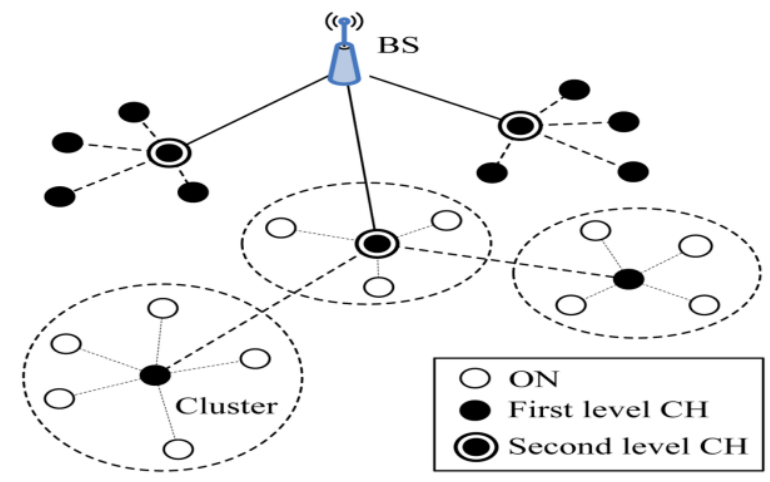

Figure 5: Clustering Topology in TEEN

\section{ADAPTIVE THRESHOLD SENSITIVE ENERGY EFFICIENT SENSOR NETWORK PROTOCOL (APTEEN):}

APTEEN aims at both capturing periodic data collections (LEACH) and reacting to time-critical events (TEEN). Thus, APTEEN is a hybrid clustering-based routing protocol that allows the sensor to send their sensed data 
periodically and react to any sudden change in the value of the sensed attribute by reporting the corresponding values to their $\mathrm{CHs}$. CHs also perform data aggregation in order to save energy. APTEEN supports three different query types namely

(i) Historical query, to analyze past data values

(ii) One-time query, to take a snapshot view of the network

(iii) Persistent queries, to monitor an event for a period of time. Energy dissipation will be lower and a large number of sensors alive in APTEEN.

\section{CONCLUSION}

One of the main challenges in the design of routing protocols for WSNs is energy efficiency due to the scarce energy resources of sensors. The energy consumption of the sensors is dominated by data transmission and reception. Therefore, routing protocols designed for WSNs should be as energy efficient as possible to prolong the lifetime of individual sensors, and hence the network lifetime. The protocols discussed have individual advantages and pitfalls. Based on the topology, the protocol and routing strategies can be applied. For realization of sensor networks, it is needed to satisfy the constraints introduced by factors such as fault tolerance, scalability, cost, topology change, environment, and power consumption. Hierarchical based routing protocols have the common objective of trying to extend the lifetime by reducing the energy consumption of the sensor network. Hierarchical based techniques have special advantage of scalability and efficient communication. Most of the routing protocols require location information for sensor nodes in wireless sensor networks to calculate the distance between two particular nodes on the basis of signal strength so that energy consumption can be estimated.

Many issues and challenges still exist that need to be solved in the sensor networks. The main objective behind the routing protocol design is to keep sensors alive as much as possible, thus prolonging the lifetime of network. For heterogeneous wireless sensor networks, many energy efficient clustering protocols are proposed which are based on residual energy, density etc. This paper is a survey of the past research works which mainly focuses on energy efficient clustering based routing protocols for wireless sensor networks and we have systematically analyzed a few classical WSN clustering routing protocols in deep. Further research would be needed to address issues related to Cluster formation, cluster head communication and data fusion etc.

Table -1 Experiment Result

\begin{tabular}{|c|c|c|}
\hline $\begin{array}{c}\text { Routing } \\
\text { protocol }\end{array}$ & QOS & Transmission scheme \\
\hline LEECH & Low & $\begin{array}{c}\text { Multi hop, cluster heads } \\
\text { directly transmit to sink }\end{array}$ \\
\hline PEGASIS & low & $\begin{array}{c}\text { Multipath, only if the } \\
\text { neighbors are at a larger } \\
\text { distance than single hop }\end{array}$ \\
\hline SAR & high & $\begin{array}{c}\text { Multi hop, Trees are } \\
\text { constructed either from node } \\
\text { to sink or sink to node }\end{array}$ \\
\hline SPEED & high & $\begin{array}{c}\text { Mutli hop, if no node } \\
\text { failures or congestions } \\
\text { Occur }\end{array}$ \\
\hline
\end{tabular}

\section{REFERENCE}

[1] S. Ran, G. Zhang, H. Gong, "Improving On Leach Protocol Of Wireless Sensor Networks Using Fuzzy Logic," J. Inf. Comput. Sci., 767$775,2010$.

[2] L.K Abdulsalam, H.M. Kamel, "W-Leach: Weighted Low Energy Adaptive Clustering Hierarchy Aggregation Algorithm For Data Streams In Wireless Sensor Networks," In Proceedings Of Ieee International Conference On Data Mining Workshops (Icdmw), Sydney, Australia, Pp. 1-8., 14 December 2010 
[3] A Comprehensive Review Of Cluster Based Energy Efficient Routing Protocols For Wireless Sensor Networks Naveen Sharma1 And Anand Nayyar2 International Journal Of Application Or Innovation In Engineering \& Management (Ijaiem)Volume 3, Issue 1, January 2014 Issn $2319-4847$

[4] Rahul Goyal, " A Review On Energy Efficient Clustering Routing Protocol In Wireless Sensor Network ", Ijret: International Journal Of Research In Engineering And Technology Eissn: 2319-1163 | Pissn: 2321-7308, Volume: 03 Issue: 06 | Jun-2014

[5] Kodali R.K., Sarma N "Energy Efficient Routing Protocols For Wsn's" International Conference On Computer Communication And Information, Pp 1-4, 4- 6 Jan, 2013.

[6] Rahman A., Anwar S. Et Al “A Survey On Energy Efficient Routing Techniques In Wireless Sensor Network" International Conference On Advanced Communication Technology, Pp 200-206,27-30 Jan,2013.

[7] Naveen Sharma1 and Anand Nayyar, " A Comprehensive Review of Cluster Based Energy Efficient Routing Protocols for Wireless Sensor Networks ", International Journal of Application or Innovation in Engineering \& Management (IJAIEM), Volume 3, Issue 1, January 2014

[8] Parminder Kaur, , Mrs. Mamta Katiyar, " International Journal of Advanced Research in Computer Science and Software Engineering ", Volume 2, Issue 11, November 2012.

[9] Swati Shamkuwar, , Prof. Vimal Shukla, " A Review on Energy Efficient Routing Protocols in Wireless Sensor Networks ", International Journal of Emerging Technology and Advanced Engineering, Volume 4, Issue 3, March 2014

[10] M. Aslam, T. Shah, N. Javaid, A. Rahim, Z. Rahman, Z. A. Khan, "CEEC: Centralized Energy Efficient ClusteringA New Routing Protocol for WSNs", 2012 9th Annual IEEE Communications Society Conference on Sensor, Mesh and Ad Hoc Communications and Networks.

[11] Mrs. Sunita S. Nandgave, " A Survey on QOS and energy efficient routing protocols in WSN", International Journal of Application or Innovation in Engineering \& Management (IJAIEM), Volume 1, Issue 2, October 2012 